\title{
Argumentación en la enseñanza de circuitos eléctricos aplicando aprendizaje activo
}

\author{
Argumentation in the teaching of electrical circuits by applying active learning \\ Esmeralda Campos ${ }^{1 @}$, Silvia Tecpan ${ }^{2}$, Genaro Zavala ${ }^{* 1,3 @}$ \\ ${ }^{1}$ Tecnologico de Monterrey, Escuela de Ingenieria y Ciencias, Monterrey, Mexico. \\ ${ }^{2}$ Universidad de Santiago de Chile, Facultad de Ciencia, Santiago, Chile. \\ ${ }^{3}$ Universidad Andres Bello, Facultad de Ingenieria, Santiago, Chile.
}

Recibida en 02 de Noviembre, 2020. Revisado en 05 de Enero, 2021. Aceptado en 09 de Enero, 2021.

\begin{abstract}
El aprendizaje de circuitos eléctricos es fundamental en la formación de ingenieros y los estudiantes tienen dificultades para comprender estos conceptos. Para ello, la investigación en educación de la física ha desarrollado estrategias que promueven argumentación y entendimiento conceptual. El objetivo de esta investigación es estudiar las dinámicas de argumentación de los estudiantes al aprender circuitos eléctricos en un curso de electricidad y magnetismo que se implementa en un ambiente de aprendizaje centrado en el estudiante con el uso de instrucción por pares y actividades de andamiaje cognitivo. La investigación se realizó por medio de una observación naturalista. Los resultados muestran que los estudiantes tienen dificultades que resuelven por medio de la argumentación. Una diferencia entre estrategias es que, durante instrucción por pares, la dinámica de discusión es en equipos grandes, mientras en las actividades de andamiaje cognitivo, es en equipos pequeños. El principal hallazgo de esta investigación es que implementar estrategias que promueven la argumentación provoca que los estudiantes logren entendimiento conceptual y desarrollen su capacidad para analizar circuitos en lugar de aplicar reglas memorizadas. Se concluye que, con estas estrategias, la argumentación sucede en cuatro niveles, la mente del estudiante, equipos pequeños, la comunidad de aprendizaje y en el grupo completo.

Palabras clave: Argumentación, aprendizaje centrado en el estudiante, instrucción por pares, andamiaje cognitivo, circuitos eléctricos, educación universitaria.
\end{abstract}

Learning electric circuits is a fundamental aspect of engineering training and students have difficulty understanding these basic concepts. For this, physics education research has developed strategies that promote argumentation and conceptual understanding. This research explores students' argumentation dynamics when learning electric circuits in an electricity and magnetism introductory course for engineering implemented in a student-centered learning environment, where Peer Instruction and Cognitive Scaffolding Activities occur. The research uses a naturalistic observation. The results show that students have difficulties and previous conceptions that, through argumentation, they modify to reach a correct conclusion. One of the main differences is that, during peer instruction, the discussion dynamics occur in groups with a larger number of students. In contrast, in cognitive scaffolding activities, the discussion dynamics occur in small teams. This research's main finding is that implementing strategies that promote argumentation causes students to achieve a deep conceptual understanding of electric circuits and develop their ability to analyze circuits rather than apply memorized rules. The conclusion is that, with these strategies, argumentation happens at four levels, in the student's mind, in small teams, in the learning community, and with the whole group.

Keywords: Argumentation, student centered learning, peer instruction, cognitive scaffolding, higher education.

\section{Introducción}

En el aprendizaje de física, el entendimiento de conceptos es un reto para estudiantes universitarios, especialmente en cursos introductorios de electricidad y magnetismo por su nivel de abstracción [1 3. Parte del proceso de aprendizaje se concentra en el contraste entre la intuición sobre un fenómeno físico con la realidad observable. Sin embargo, dado que los fenómenos de electricidad y magnetismo no son directamente observables,

\footnotetext{
* Correo electrónico: genaro.zavala@tec.mx
}

los estudiantes no han desarrollado una intuición al respecto, lo que se evidencia a través de concepciones alternativas y dificultades de aprendizaje 4.

El tema de circuitos eléctricos forma parte de la mayoría de los cursos introductorios de electricidad y magnetismo de carreras de ingeniería a nivel universitario. Es un conocimiento necesario, dado que la electricidad es parte esencial de la vida moderna en actividades de uso cotidiano como iluminación, calefacción, refrigeración, computadoras, transportación pública, es decir, con diversas aplicaciones [5, 6]. En la educación preuniversitaria en México, algunos estudiantes tienen 
acercamiento al tema de circuitos eléctricos; sin embargo, los resultados de diversas investigaciones sugieren que los estudiantes universitarios no necesariamente han desarrollado un entendimiento conceptual profundo sobre las nociones básicas, como corriente, resistencia y diferencia de potencial, debido a una falta de relación con la electrostática 2. Además, se ha encontrado que incluso los docentes preuniversitarios sostienen concepciones alternativas respecto a la electricidad y magnetismo lo que sugiere que pueden transmitir y reforzar concepciones erróneas entre sus estudiantes [7]. En este contexto se ha encontrado que las dificultades para comprender los fenómenos electromagnéticos persisten en los estudiantes de cursos introductorios de física a nivel universitario [2, 8, 11].

La literatura sobre el aprendizaje de las ciencias sugiere que la argumentación ayuda a los estudiantes a mejorar su razonamiento científico y promover $\mathrm{su}$ entendimiento conceptual 12 14. Actualmente, la educación científica ha transitado hacia el desarrollo de habilidades de pensamiento y prácticas asociadas a la investigación científica más que a la memorización, una práctica científica es la argumentación [12, 15. De acuerdo con Osborne [14], la argumentación promueve el entendimiento conceptual [16], ya que los estudiantes se ven en la necesidad de justificar sus conclusiones, las cuales pueden ser desafiadas por otras ideas. Al contrastar ideas, los estudiantes tienen la oportunidad de evaluar sus propias concepciones y aprender nuevas, favoreciendo así la construcción y asimilación de nuevos conceptos [17.

El objetivo de esta investigación es explorar las dinámicas de argumentación de los estudiantes al aprender circuitos eléctricos en un curso introductorio de electricidad y magnetismo para ingeniería que se implementa en un ambiente de aprendizaje centrado en el estudiante, donde se aplican estrategias de instrucción por pares y actividades de andamiaje cognitivo. En concreto, se busca (1) analizar momentos donde, por medio de la argumentación, los estudiantes se enfrenten a sus propias concepciones e ideas previas, (2) analizar cualitativamente cómo estos momentos contribuyen al aprendizaje y (3) distinguir las características entre las discusiones que surgen durante las diferentes estrategias didácticas. La investigación es relevante para el profesorado de ciencia que busca incluir la argumentación en su práctica docente con el fin de mejorar el aprendizaje de los estudiantes y para la línea de investigación de la argumentación en el aprendizaje de la ciencia. Resulta importante distinguir las características entre las discusiones que surgen en ambas estrategias didácticas, ya que se ha observado que el ambiente donde se realiza la investigación promueve la formación de comunidades de aprendizaje que son diferentes para las dos estrategias propuestas [18. Identificar estas diferencias puede ser de utilidad para la toma de decisiones de los profesores de ciencias al formar grupos pequeños en el aula y para incluir una variedad de estrategias didácticas de aprendizaje colaborativo.

\section{Revisión de la Literatura}

Se identifican tres componentes principales de la investigación: (a) dificultades para el aprendizaje de circuitos eléctricos, (b) la argumentación en el aprendizaje de ciencias y (c) estrategias didácticas que promueven la argumentación. Se identifica el ambiente de aprendizaje centrado en el estudiante como contexto de la investigación. A continuación, se presenta una breve revisión de la literatura relevante para la presente investigación.

\subsection{Dificultades en el aprendizaje de circuitos eléctricos}

El aprendizaje de circuitos eléctricos en nivel universitario es un aspecto fundamental de la formación de ingenieros por sus aplicaciones en el desarrollo tecnológico [19. Sin embargo, los estudiantes tienen dificultades para comprender los conceptos básicos que explican el comportamiento de los circuitos eléctricos, entre ellas: (1) Los focos (o resistencias) gastan corriente en el circuito; (2) la batería es una fuente constante de corriente, y (3) tanto la dirección de la corriente, como el orden y la colocación física de los elementos son importantes [20]. Adicionalmente, muchos estudiantes tienen dificultades para comprender el concepto de resistencia equivalente y su relación con el brillo de los focos en un circuito de corriente directa 20]. La recurrencia de estas dificultades se ha observado en estudios recientes 21. Andrea, Barbosa, Silveira y Santos [21] sugieren que la dificultad de los estudiantes al pensar que la corriente se gasta al pasar por una resistencia se debe a una relación con el gasto de energía.

Por otra parte, se ha documentado que las dificultades en la comprensión de la Ley de Ohm y el comportamiento de los circuitos de corriente continua (CC) pueden deberse al hecho de que los circuitos eléctricos se tratan con poca o nula vinculación a otros temas del electromagnetismo, particularmente a la circulación del campo eléctrico 2. En otro estudio que destaca la importancia del campo eléctrico en el aprendizaje de circuitos eléctricos se analizó el razonamiento causal que utilizan los estudiantes universitarios para explicar cómo funcionan los circuitos de CC. Se encontró que existe diferencia entre la comprensión macroscópica y microscópica del fenómeno. Desde la perspectiva macroscópica, que es la más utilizada por los estudiantes, se utiliza un razonamiento relacional en el que se incluye la conservación de la corriente eléctrica, mientras que sólo una minoría de estudiantes es capaz de proponer explicaciones a nivel microscópico (cargas o electrones) con razonamiento unidireccional de causa-efecto [22]. 


\subsection{Argumentación en el aprendizaje de ciencias}

A lo largo de la historia, la ciencia se ha construido por medio de la argumentación entre científicos. Al definir la argumentación como una práctica social, se logra cambiar la concepción de la naturaleza de la ciencia, pues el conocimiento científico se concibe como una construcción social [13. De acuerdo con JiménezAleixandre, Álvarez y Lago [23], la construcción de la ciencia no se limita a hacer experimentos, sino también incluye discutir ideas, evaluar diferentes alternativas y elegir entre distintas explicaciones para un fenómeno. El discurso colaborativo y la argumentación promueven el aprendizaje, ya que para aprender los estudiantes necesitan la oportunidad de desarrollar conclusiones, justificar las ideas que tienen y desafiarlas [14. La argumentación dialógica ayuda a desarrollar el pensamiento científico y promover el aprendizaje de los estudiantes. En este sentido, la argumentación es un proceso crucial para aprender a pensar y construir nuevos conceptos; cuando sus concepciones son desafiadas, los estudiantes tienen la oportunidad de entender por qué ciertas ideas son incorrectas mientras otras son correctas y, así evaluar esas mismas concepciones para aprender nuevas ideas.

En la investigación en la enseñanza de las ciencias, se ha utilizado el modelo de argumentación de Toulmin [24] para analizar las discusiones de los estudiantes e identificar los elementos de argumentación y las relaciones entre ellos. Dentro del modelo de argumentación de Toulmin, los elementos básicos de los argumentos son los datos, las conclusiones, las justificaciones y el conocimiento base. En la literatura también se identifican estos elementos como aserción, datos y garantías [25]. Los elementos secundarios son los calificadores modales y la refutación [13. Los elementos que conforman un argumento de acuerdo con este modelo se resumen en la Tabla 1.

Bertelle, Rocha y Domínguez-Castiñeiras [26] utilizaron este modelo para analizar las discusiones de los estudiantes durante un laboratorio sobre equilibrio químico; el modelo les sirvió para identificar los elementos de los argumentos y las relaciones entre ellos. García-Rodeja Gayoso y Sesto Varela [27] analizaron las pruebas que dan los estudiantes para la construcción de explicaciones científicas por medio del patrón de argumentación de
Toulmin, y encontraron que los estudiantes son selectivos respecto a las pruebas que utilizan. Molina et al. [25] utilizaron el modelo de argumentación de Toulmin para analizar la estructura y la dinámica de las discusiones de los estudiantes en el aprendizaje de geometría del espacio en un contexto de matemáticas.

\subsection{Estrategias didácticas que promueven la argumentación}

En la investigación de la educación de la física se han desarrollado estrategias didácticas que promueven la argumentación. Se presenta la revisión de literatura de dos estrategias que son relevantes para el estudio: instrucción por pares [28] y actividades de andamiaje cognitivo [29]. Ambas estrategias didácticas son de aprendizaje activo y colaborativo, siguiendo las definiciones de Prince 30, porque involucran al estudiante en su proceso de aprendizaje y trabajan en equipos pequeños hacia una meta común. Los integrantes del equipo aportan ideas utilizando la argumentación oral para construir un entendimiento compartido del problema que analizan.

Instrucción por pares se refiere a la estrategia didáctica propuesta por Mazur y Hillborn [28], donde los estudiantes discuten para resolver una situación conceptual. El profesor plantea un problema conceptual en forma de pregunta de opción múltiple a los estudiantes y ellos escogen una respuesta de manera individual. Después de esto, el profesor les invita a encontrar a alguien que haya escogido una respuesta diferente y discutir argumentando por qué escogieron esa respuesta. Tras la discusión, los estudiantes escogen nuevamente una respuesta de manera individual, que puede ser diferente a la respuesta que hayan escogido antes de discutir. Mazur y Hillborn [28] proponen que los estudiantes que han escogido la respuesta correcta tienden a poseer argumentos más fuertes sobre su respuesta y terminan convenciendo a sus compañeros de la respuesta correcta.

Esta estrategia ha sido ampliamente aplicada en la investigación de la educación de la física [31 33] y específicamente en el aprendizaje de circuitos eléctricos en contextos de aprendizaje activo [34]. Esta estrategia ha demostrado promover el entendimiento conceptual y las habilidades de solución de problemas en estudiantes

Tabla 1: Elementos del modelo de argumentación de Toulmin.

\begin{tabular}{lll}
\hline Tipo & \multicolumn{1}{c}{ Elemento } & \multicolumn{1}{c}{ Descripción } \\
\hline Básico & Conclusión & El enunciado que se pretende validar \\
& Datos & Hechos que apoyan la conclusión \\
& Justificaciones & Razones que justifican la relación entre datos y conclusión \\
& Conocimiento básico & Supuestos que respaldan las justificaciones \\
\multirow{2}{*}{ Secundario } & Calificador modal & Especifican condiciones y limitaciones de la conclusión \\
& Refutación & Especifican condiciones bajo las cuales la conclusión no se cumple \\
\hline
\end{tabular}


de física introductoria 35]. Esta estrategia permite a los estudiantes alcanzar un entendimiento conceptual más profundo para justificar mejor sus argumentos [31]. Los estudiantes que tienen la respuesta correcta han pasado por un proceso para comprender el tema recientemente, por lo que tienden a reconocer mejor las dificultades de sus compañeros. Además, el lenguaje que se utiliza entre pares es distinto al que utiliza el profesor, lo que facilita que los estudiantes con dificultades las superen. La instrucción por pares ofrece a los estudiantes una oportunidad para construir conocimiento en conjunto [32.

Una forma alternativa de diseñar actividades de aprendizaje es utilizar lo que se ha llamado andamiaje cognitivo [36] o simplemente andamiaje [37. Parafraseando a Wood et al. [37, el andamiaje es cualquier proceso que permite al estudiante alcanzar una comprensión de una concepción científica, resolver un problema, llevar a cabo una tarea, o lograr una meta que sería muy difícil alcanzar sin ayuda. El andamio cognitivo incluye estrategias (por medio de preguntas) que ayudan a los estudiantes a reflexionar, pensar o conceptualizar 36. Las actividades de andamiaje cognitivo [29] son actividades de aprendizaje colaborativo diseñadas con base en la investigación de la educación de la física, donde diversos profesores han utilizado el andamio cognitivo por algunos años [38 44].

Las actividades de andamiaje cognitivo tienen el formato de tutoriales, inspiradas en los propuestos por McDermott y Shaffer [45]; se realizan en hojas de trabajo de manera escrita y la discusión es oral. Los estudiantes trabajan colaborativamente, intercambiando ideas y razonamientos para responder a las preguntas planteadas. El equipo docente supervisa el desempeño de los estudiantes haciendo preguntas a cada equipo y orientando hacia el logro del objetivo de la actividad. Al final realiza un plenario como cierre de la actividad, revisando los conceptos clave a los que debieron haber llegado los estudiantes, reafirmando el conocimiento adquirido. Las secuencias de actividades de andamiaje cognitivo utilizadas en este estudio fueron diseñadas con base en la investigación en enseñanza de la física, lo que concuerda con las propuestas de Guisasola, Garmendia, Montero y Barragués [46] acerca del diseño de secuencias de enseñanza.

\subsection{Contexto de la investigación: ambiente de aprendizaje centrado en el estudiante}

El ambiente de aprendizaje donde se desarrolla la clase puede ser otro elemento que contribuye a que suceda la argumentación entre pares. En el Aprendizaje Centrado en el Estudiante (ACE) el estudiante toma un papel central en la construcción de conceptos por medio de actividades colaborativas, uso de tecnología y métodos de aprendizaje activo, promoviendo la argumentación entre pares [47. El ambiente SCALE-UP (por sus siglas en inglés), provee una infraestructura adecuada para promover el ACE (http://scaleup.ncsu.edu/) con características específicas que pueden adaptarse a las necesidades de cada universidad. En México, se realizó la primera adaptación del ambiente SCALE-UP, llamada sala ACE, en una universidad privada al norte del país, la cual cuenta con ocho mesas circulares para nueve personas cada una; una mesa está diseñada para apoyar la investigación educativa, cuenta con tres videocámaras y tres micrófonos para facilitar la grabación de las interacciones entre los estudiantes que ocupan esa mesa [47.

Las salas estilo auditorio, donde el instructor tiene un papel central, se han utilizado para aplicar la estrategia didáctica de instrucción por pares [33]. Particularmente, en el tema de circuitos eléctricos se han analizado las discusiones que surgen con la instrucción por pares en contextos de aprendizaje activo [34, pero no se han realizado comparaciones de estas discusiones al situarse en ambientes de aprendizaje centrado en el estudiante. En el presente estudio, surge la necesidad de explorar el aprendizaje sobre circuitos eléctricos a través de las dinámicas de argumentación de los estudiantes en un curso introductorio de electricidad y magnetismo que se implementa en un ambiente de aprendizaje centrado en el estudiante, donde se aplican estrategias de instrucción por pares y actividades de andamiaje cognitivo.

\section{Metodología}

La investigación se realizó con un método cualitativo fenomenológico ya que se basa en observación naturalista [48]. Se observaron dinámicas de discusión en una clase introductoria de electricidad y magnetismo con 37 estudiantes de física en la sala de aprendizaje centrado en el estudiante, donde la argumentación ocurre de manera natural, en una universidad privada al norte de México. En este curso, las clases son de tres horas semanales e incluyen: presentación teórica por parte del profesor, instrucción por pares y actividades de andamiaje cognitivo. Además, se realiza una sesión de laboratorio de 90 minutos con los tutoriales para física introductoria 45]. Los estudiantes tienen evaluaciones de lectura cada semana del libro de texto de física introductoria apto para ingeniería [49]. Se aplica un inventario conceptual al inicio y al final del semestre.

Los estudiantes de este curso no reciben instrucción explícita sobre los elementos que componen un argumento, sin embargo, se les invita constantemente a justificar sus aseveraciones cuando hay acuerdos y desacuerdos entre los miembros del equipo, promoviendo activamente que la argumentación surja de manera natural. En una sesión de clase cotidiana, se dedican entre 30 y 60 minutos a la dinámica de instrucción por pares, donde se presentan entre cinco y diez preguntas conceptuales. Cada pregunta conceptual puede tener discusiones de 3 a 5 minutos. En cuanto a las actividades de andamiaje cognitivo, en cada sesión se procura tener al menos tres actividades, cada una con una duración de 10 a 20 minutos. Por lo que también se le dedica entre 30 
y 60 minutos a este tipo de dinámica. Estas cantidades varían y se adecuan al tema de clase y a la respuesta de los estudiantes.

A partir de los resultados de la primera evaluación parcial del curso, después de concluir los temas de electrostática y antes de comenzar circuitos eléctricos, se formaron equipos de trabajo para todos los estudiantes. Los equipos de trabajo se formaron con tres estudiantes, siguiendo los criterios para la formación de grupos pequeños de trabajo en el aula [50. Cada equipo incluyó a un estudiante con nota promedio a partir de los resultados de la evaluación de medio término, un estudiante con nota arriba del promedio y un estudiante con nota por debajo del promedio, de manera que las interacciones entre ellos permitan la colaboración entre estudiantes con mayor heterogeneidad entre sus perfiles académicos 51. Los equipos se asignaron de manera aleatoria a las diferentes mesas de la sala. Cada mesa está conformada por tres equipos de tres personas. Los participantes de este estudio son nueve estudiantes ubicados en la mesa equipada para investigación de la sala ACE, en total siete hombres y dos mujeres.

El instrumento principal de la investigación es la observación realizada por los investigadores por medio del equipo técnico que se encuentra en la sala ACE. Para consideraciones éticas, al inicio del curso se les comunicó a los estudiantes que el aula está dedicada a la investigación y que podrían ser grabados en cualquier momento. Todos los estudiantes del curso firmaron un consentimiento informado sobre su participación en la investigación en el que se estableció su participación voluntaria y que no afecta su evaluación en el curso. El equipo técnico incluye tres cámaras y tres micrófonos de grabación que permitieron analizar a detalle y fuera de tiempo real las interacciones de los estudiantes. Se realizaron grabaciones de las interacciones entre los estudiantes en las cinco sesiones del curso que corresponden al tema de circuitos eléctricos, con una duración total de 13:40 horas (sesiones 7 a 11, de un total de 16 sesiones). Se grabaron a los tres diferentes equipos de la mesa de investigación de la sala ACE en intervalos iguales de tiempo durante el horario de clase establecido. Las grabaciones fueron realizadas con cámaras instaladas en el techo, visibles, pero con función oculta de tal manera que los estudiantes no saben si están siendo grabados. Esto facilita que las interacciones ocurran de manera natural.

Ya que se obtuvo cada grabación, se reprodujeron los videos en búsqueda de momentos que mostraran argumentación entre los integrantes del equipo o de la mesa y se analizaron estas discusiones. Los investigadores del estudio realizaron transcripciones de los momentos identificados utilizando un software para la reproducción de video. En las transcripciones, se identifica a los estudiantes por medio de etiquetas para mantener su confidencialidad. En la Figura 1 se muestran las etiquetas de los estudiantes y su ubicación en la mesa de investigación.

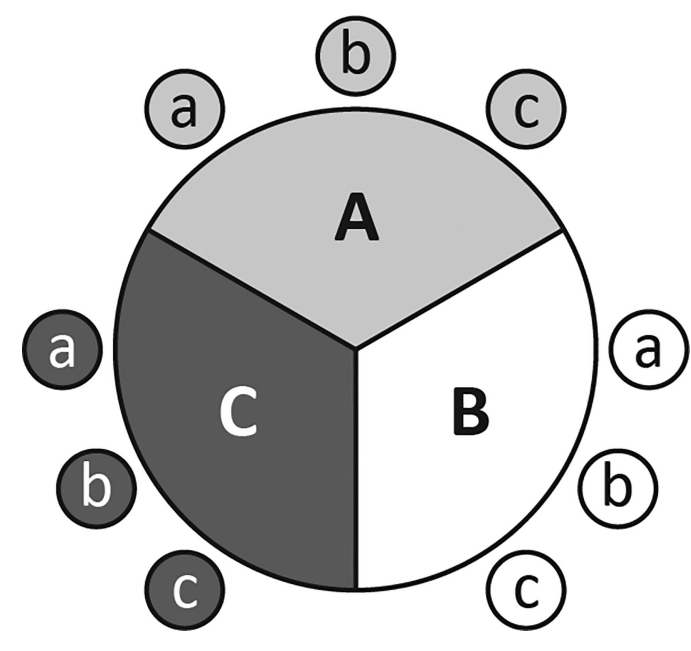

Figura 1: Organización habitual de los estudiantes por equipos de la mesa de investigación. Se utilizan las etiquetas $\mathrm{Aa}-\mathrm{Cc}$ para identificar a los estudiantes.

Tabla 2: Los participantes se identifican por etiquetas y se describe la participación que cumplen en la construcción de argumentos. Las etiquetas $\mathrm{Aa}-\mathrm{Cc}$ se utilizan para identificar a los estudiantes de acuerdo con su distribución habitual alrededor de la mesa (ver Figura 1).

\begin{tabular}{ll}
\hline Estudiante & Descripción de su participación en la mesa \\
\hline $\mathrm{Aa}$ & $\begin{array}{l}\text { Fundamenta sus conclusiones con } \\
\text { datos y refuta las conclusiones }\end{array}$ \\
$\mathrm{Ab}$ & $\begin{array}{l}\text { Hace conclusiones iniciales, } \\
\text { estableciendo la argumentación }\end{array}$ \\
$\mathrm{Ac}$ & $\begin{array}{l}\text { Contribuye con opiniones o } \\
\text { cuestionamientos, escucha } \\
\text { activamente }\end{array}$
\end{tabular}

$\mathrm{Ba} \quad$ Hace conclusiones iniciales, y hace uso extensivo de calificadores modales

$\mathrm{Bb} \quad$ Hace cuestionamientos y refutaciones

Bc Respalda sus conclusiones con datos, justificaciones, fórmulas y procedimientos

Ca Contribuye con opiniones y cuestionamientos, escucha activamente

$\mathrm{Cb} \quad$ Hace cuestionamientos y escucha activamente

Cc Hace conclusiones iniciales, respalda sus conclusiones con datos y justificaciones

A petición del equipo docente, los estudiantes se ubican en el mismo lugar consistentemente durante el curso. En la Tabla 2 se describe la participación que toman los participantes en las discusiones y se identifican por medio de las etiquetas expuestas en la Figura 1 
Como se observa en la Tabla 2 se describe la participación de los estudiantes con base en sus aportaciones a las discusiones y la generación de argumentos. Estas descripciones se basan en los elementos del modelo de argumentación de Toulmin. Se puede observar que en el equipo A, los estudiantes Aa y Ab contribuyen a la argumentación por medio de conclusiones, datos y refutaciones, mientras el estudiante Ac realiza cuestionamientos o comparte sus opiniones. En el equipo B, los tres estudiantes aportan elementos argumentativos, como conclusiones, calificadores modales, refutaciones, datos y justificaciones. En el equipo C, el estudiante Cc aporta elementos argumentativos, como conclusiones, datos y justificaciones, mientras sus compañeros $\mathrm{Ca}$ y $\mathrm{Cb}$ hacen cuestionamientos y escuchan activamente. Se resalta que algunos estudiantes pueden tener una comprensión correcta de los temas de circuitos eléctricos, aunque decidan no contribuir a las discusiones o argumentos. Se considera que la escucha activa de las discusiones que suceden entre los compañeros es un elemento adicional que promueve la argumentación.

\subsection{Análisis de datos y validación}

Se analizaron las discusiones de los estudiantes en dos estrategias didácticas: instrucción por pares y actividades de andamiaje cognitivo. Para el análisis de datos, se utilizó el modelo de argumentación de Toulmin [24] para verificar si sus discusiones cumplían con los elementos básicos que deben estar presentes en cualquier argumento para que sea considerado como tal y elementos secundarios que agregan complejidad al argumento. Como estrategia de análisis de datos, se identificaron diferentes momentos donde ocurrieron discusiones durante los dos tipos de actividades. Se transcribieron las discusiones, identificando a los estudiantes por medio de etiquetas. A partir de las transcripciones, uno de los investigadores clasificó las contribuciones a la discusión respecto a los elementos del modelo de argumentación de Toulmin.

La clasificación fue validada por tres investigadores, dos expertos que forman parte de la investigación y un investigador experto externo. La credibilidad de los hallazgos cualitativos se realizó a través de la estrategia peer debriefing que consistió en someter el diseño, planeación y resultados del estudio al escrutinio de un colega familiarizado con el tipo de investigación realizada, pero ajeno al proyecto de investigación [52, 53].

\section{Resultados}

Para presentar los resultados, se identifican las dinámicas de discusión y de argumentación para cada estrategia didáctica. Se identificaron los elementos de argumentación de Toulmin durante las discusiones. Este análisis permitió tener un panorama sobre la complejidad de los argumentos: si el argumento incluye elementos secundarios, implica que su complejidad es mayor. Primero se presenta la transcripción de una discusión durante instrucción por pares, a manera de ejemplo, y se resaltan momentos de esta donde es posible observar de manera cualitativa que hay cambio en la comprensión de los conceptos, dando la oportunidad de aprendizaje. Posteriormente, se presenta el ejemplo de una discusión que surgió durante una de las actividades de andamiaje cognitivo. Como parte del análisis, se encontraron de manera cualitativa momentos clave de aprendizaje durante esta actividad. A partir del análisis realizado para cada tipo de actividad, se plantean observaciones sobre sus características. En la sección de discusión, se comparan las diferencias entre las características identificadas.

\subsection{Argumentación sobre circuitos eléctricos durante instrucción por pares}

Durante la clase de circuitos sobre corriente y resistencia, se plantearon diversas situaciones a manera de pregunta conceptual, donde era necesario que los estudiantes analizaran circuitos con diferentes arreglos de baterías y resistencias con conexiones en serie y/o en paralelo. Estas preguntas aumentan de dificultad gradualmente y son progresivas para la construcción del concepto. La primera situación presenta un circuito con dos resistencias conectadas en serie a una batería. Se les indica que la corriente que pasa por la primera resistencia es $I$ y se les pregunta cómo es la corriente que pasa por la segunda resistencia. Las opciones fueron: (a) Igual a $I$, (b) La mitad de $I$, (c) Menor que $I$ pero no necesariamente la mitad, (d) El doble que $I$, y (e) Mayor que $I$ pero no necesariamente el doble. En la Tabla 3 se presenta una discusión que surgió durante esta actividad.

Después de esta situación, se cubrieron varios arreglos diferentes de circuitos cuyo objetivo es esclarecer el comportamiento de la corriente y el voltaje de los diferentes elementos (batería y resistencias) en los circuitos conectados en serie y en paralelo. Posteriormente, se les planteó a los estudiantes una situación donde se tiene una bombilla que pertenece a un circuito y por el cual fluye una corriente. Se conecta un cable uniendo las dos terminales de la bombilla. Con este alambre conectado se les dan a los estudiantes las siguientes opciones: (a) ahora toda esta corriente circula a través de la bombilla, (b) ahora la mitad de esta corriente se va a través de la bombilla y la mitad a través del alambre y (c) ahora toda esta corriente fluye a través del alambre. Se les pidió a los estudiantes votar de manera individual y, tras obtener los resultados, discutir con sus compañeros sobre sus respuestas. La indicación para discutir es que si encuentran a alguien con quien no estén de acuerdo, le den argumentos para convencerlo de su respuesta, pero si resulta que todos están de acuerdo, que argumenten por qué respondieron de esa manera. El argumento que resultó de este ejercicio se presenta en la Tabla 4 .

Del análisis de los argumentos presentados en la Tabla 3 y la Tabla 4, usando el modelo de Toulmin, se observa que se incluyen los cuatro elementos básicos: conclusiones, justificación, datos y conocimiento básico. 
Tabla 3: Argumento durante la dinámica de instrucción por pares donde los estudiantes discuten sobre la corriente que pasa por dos resistencias conectadas en serie a una batería.

\begin{tabular}{lcll}
\hline Línea & Miembro & \multicolumn{1}{c}{ Aseveración } & Elemento \\
\hline 1 & $\mathrm{Ab}$ & Es C porque el voltaje es el único que queda igual, ¿o es al revés? & Conclusión \\
2 & $\mathrm{Ab}$ & ¿Es la corriente la que se queda igual y el voltaje cambia? & \\
3 & $\mathrm{Aa}$ & Ajá, el voltaje sí cambia. & \\
4 & $\mathrm{Ab}$ & OK, entonces me había confundido con eso & \\
5 & $\mathrm{Ba}$ & ¿Por qué cambia? & Refutación \\
6 & $\mathrm{Ab}$ & Porque es más resist ... o sea pasa por resistencia & Justificación \\
7 & $\mathrm{Ba}$ & ¿y qué hace la resistencia? & \\
8 & $\mathrm{Aa}$ & si las resistencias son iguales en sí el voltaje es igual pero si las & Justificación, \\
& & resistencias cambian, el voltaje es cuando cambia & Conocimiento básico \\
9 & $\mathrm{Ab}$ & Mm & Conocimiento básico \\
10 & $\mathrm{Aa}$ & Y la corriente siempre es igual, no importa las resistencias & Refutación \\
11 & $\mathrm{Ac}$ & Y si la resistencia no es igual, ¿da lo mismo? & \\
12 & $\mathrm{Ba}$ & ¿Qué? & Conclusión \\
13 & $\mathrm{Ac}$ & ¿Si las resistencias no son idénticas? & \\
14 & $\mathrm{Aa}$ & Da igual, mientras estén en serie la corriente es la misma &
\end{tabular}

Se observa también que los estudiantes tienden a incluir refutaciones y cuestionamientos que agregan complejidad a su argumentación. El movimiento entre estos elementos no es lineal, sino que los estudiantes pueden utilizar los elementos del argumento para llegar a una conclusión, o bien partir de una conclusión para hacer justificaciones, datos y refutaciones. El análisis de este y de otros episodios de argumentación durante instrucción por pares sugiere que estos procesos argumentativos giran alrededor de las conclusiones.

En la discusión de la Tabla 3 se presenta en la línea 1 una conclusión incorrecta sobre la corriente que pasa por la segunda resistencia. El estudiante menciona que el voltaje es el mismo para las dos resistencias. Sin embargo, inmediatamente se cuestiona sobre su aseveración y admite que se había confundido sobre ese concepto en la línea 4 . En las líneas 5 y 6 , el estudiante Ba indaga sobre la confusión de Ab, a lo que Ab responde que la corriente pasa por la resistencia implicando que la corriente se gasta al pasar por la resistencia. Esta concepción es una dificultad recurrente en el aprendizaje de circuitos eléctricos [20, 54 que puede indicar que los estudiantes relacionan la corriente con la energía [21. En las líneas 8 y 10, se involucra el estudiante Aa dando una justificación que se basa en conocimiento básico sobre el comportamiento de los circuitos en serie. El estudiante Ac le hace cuestionamientos sobre las resistencias, para lo que Aa concluye que mientras las resistencias estén conectadas en serie, la corriente que pase a través de ellas es la misma, independientemente del valor de las resistencias. Se observa cómo los estudiantes partieron de una conclusión incorrecta y por medio de justificaciones, conocimiento básico y refutaciones, lograron llegar a una conclusión correcta para el circuito mostrado. Aunque la dificultad conceptual puede persistir, se observa que la argumentación brinda una oportunidad para que los estudiantes confronten sus concepciones previas.

En la discusión de la Tabla 4, se muestran dos argumentos que suceden a nivel individual y que los estudiantes exponen a sus compañeros pensando en voz alta, las líneas 1 y 16 . En la línea 1, el estudiante Ba expone su justificación y conclusión. Es un argumento correcto, pero es sencillo porque el estudiante parece estar aplicando una regla sugerida por la ley de Ohm, a menor resistencia mayor corriente. El estudiante $\mathrm{Ab}$ se muestra confundido y cuestiona el enunciado de Ba, lo que le permite a este último elaborar más por medio de datos (líneas 2 a 9). En la línea 10, el estudiante Aa comienza a dudar sobre lo que se ha dicho; en la línea 16, parte de la ley de Ohm para hacer un argumento más complicado, ya que además de pensar en la relación entre corriente y resistencia, involucra al voltaje. El estudiante comete un error al hacer sus relaciones mentales en voz alta y llega a una conclusión incorrecta. En las líneas posteriores $\mathrm{Ab}$, Ba y Cc cuestionan la conclusión de Aa, lo que fomenta que el mismo estudiante se dé cuenta de su error y cambie su conclusión. La participación de los estudiantes que cuestionan los enunciados de sus compañeros enriquece la discusión porque permite que 
Tabla 4: Argumento durante la dinámica de instrucción por pares donde los estudiantes discuten sobre lo que pasa con la corriente cuando se conecta un cable a las dos terminales de una bombilla.

\begin{tabular}{|c|c|c|c|}
\hline Línea & Miembro & Aseveración & Elemento \\
\hline$\overline{1}$ & $\mathrm{Ba}$ & $\begin{array}{l}\text { Lo que yo estoy viendo es que la bombilla se puede comportar como } \\
\text { unas resistencias y el hecho de puentearlo es como poner una } \\
\text { resistencia cero entonces la corriente tiende a ir a donde no haya } \\
\text { resistencia o donde haya menos resistencia y entonces o sea va a ir } \\
\text { toda la corriente por el puente, porque trata de evitar constantemente } \\
\text { la resistencia }\end{array}$ & $\begin{array}{l}\text { Justificación y } \\
\text { conclusión }\end{array}$ \\
\hline 2 & $\mathrm{Ba}$ & Eh toda la corriente fluye a través del alambre & \\
\hline 3 & $\mathrm{Ab}$ & Pero no sería toda, ¿no? Sería parte & Refutación \\
\hline 4 & $\mathrm{Ba}$ & No, absolutamente toda & \\
\hline 5 & $\mathrm{Ba}$ & $\begin{array}{l}\text { Si fuera una resistencia abajo, menor, ahí se distribuye } \\
\text { proporcionalmente }\end{array}$ & \\
\hline 6 & $\mathrm{Ab}$ & Ah ok & \\
\hline 7 & $\mathrm{Ba}$ & Pero aquí como es un alambre & \\
\hline 8 & $\mathrm{Ab}$ & No hay & \\
\hline 9 & $\mathrm{Ba}$ & $\begin{array}{l}\text { Un alambre se considera que es un conductor con resistencia ideal } \\
\text { cero, aunque originalmente supuestamente es } 0.000001 \text { de resistencia y } \\
\text { entonces así, en todo caso si fuera } 2 \text { ohm y } 3 \mathrm{ohm} \text {, seguiría en } \\
\text { proporción }\end{array}$ & Datos \\
\hline 10 & $\mathrm{Aa}$ & Pero & \\
\hline 11 & $\mathrm{Ba}$ & $\begin{array}{l}\text { Pero más al } 2 \text { ohm, porque es menor resistencia, se quedaría más } \\
\text { corriente acá que acá }\end{array}$ & Conclusión \\
\hline 12 & $\mathrm{Aa}$ & Uhm & \\
\hline 13 & $\mathrm{Ba}$ & ¿Qué pasó? & \\
\hline 14 & $\mathrm{Aa}$ & $\begin{array}{l}\text { Espera, creo que no, o si el voltaje es, pon tú, la corriente por la } \\
\text { resistencia }\end{array}$ & Conocimiento básico \\
\hline 15 & $\mathrm{Ba}$ & ¿Cómo? & \\
\hline 16 & $\mathrm{Aa}$ & $\begin{array}{l}\text { El voltaje es la corriente por la resistencia, el voltaje es constante, la } \\
\text { resistencia es muy chica a comparación de la otra, o sea a } \\
\text { comparación de la de la bombilla entonces la corriente o sea tiene que, } \\
\text { o sea si tienes la misma corriente multiplicada por } .5 \text { que así la tienes } \\
\text { multiplicada por } 2 \text {, la más chica te da un, tienes que tener una } \\
\text { corriente más chica }\end{array}$ & $\begin{array}{l}\text { Datos, justificación y } \\
\text { conclusión }\end{array}$ \\
\hline 17 & $\mathrm{Ab}$ & Pero eso ¿por qué? O sea ¿por la fórmula? & \\
\hline 18 & $\mathrm{Ba}$ & Pero ¿por qué se parte la corriente? & \\
\hline 19 & $\mathrm{Aa}$ & ¿Cómo? & \\
\hline 20 & $\mathrm{Ba}$ & O sea, no tendría & \\
\hline 21 & $\mathrm{Aa}$ & $\begin{array}{l}\text { Sí, si se va a partir la corriente, pero o sea creo que va más hacia la } \\
\text { bombilla que hacia la otra }\end{array}$ & \\
\hline 22 & $\mathrm{Cc}$ & No & \\
\hline 23 & $\mathrm{Ba}$ & Hacia la bombilla no & Refutación \\
\hline 24 & $\mathrm{Cc}$ & Es que la bombilla & \\
\hline 25 & $\mathrm{Aa}$ & Ah no porque tiene menos resistencia, el otro & Justificación \\
\hline 26 & $\mathrm{Ba}$ & La caída de voltaje es más difícil de romper & Justificación \\
\hline
\end{tabular}


se elabore más el argumento e incluso que se cambie de conclusión, lo que evidencia que puede ocurrir el aprendizaje.

Se puede observar de los argumentos presentados en la Tabla 3 y la Tabla 4 que los estudiantes que principalmente interactúan son $\mathrm{Aa}, \mathrm{Ab}$, Ba y Cc. La interacción se da entre estudiantes pertenecientes a los tres equipos pequeños que conforman la mesa. Esta dinámica de argumentación se observó de manera continua en los episodios de instrucción por pares. Este resultado sugiere que en instrucción por pares la discusión se concentra en la mesa y no por equipo, creando una comunidad de aprendizaje facilitada por la infraestructura del aula [47. Se describe a una comunidad de aprendizaje como un grupo de personas de distintos niveles de especialidad con la meta grupal de expandir el conocimiento de la comunidad [55]. En este caso, la comunidad de aprendizaje es de nueve personas.

\subsection{Ciclos de argumentación durante actividades de andamiaje cognitivo}

Durante la clase de circuitos sobre corriente y resistencia, los estudiantes resolvieron una actividad de andamiaje cognitivo donde se les presentó el circuito de la Figura 2. Se les indicó que no se conocen los valores de las resistencias ni el valor relativo entre ellas. Se les pidió a los estudiantes ordenar de mayor a menor tanto la corriente como las diferencias de potencial de todos los elementos del circuito (batería y resistencias). Por motivos de extensión, no se presenta el argumento que surgió al analizar este circuito, sino que se describen cuatro fragmentos clave para este análisis. Los estudiantes que participan en este diálogo son Ba, Bb y Bc (ver Tabla 2).

Inicialmente, los estudiantes discutieron sobre cómo sería la resistencia equivalente de la sección en paralelo del circuito; específicamente, si la resistencia equivalente de la sección en paralelo (R2 y R3) sería menor que la resistencia que se encuentra conectada en serie con

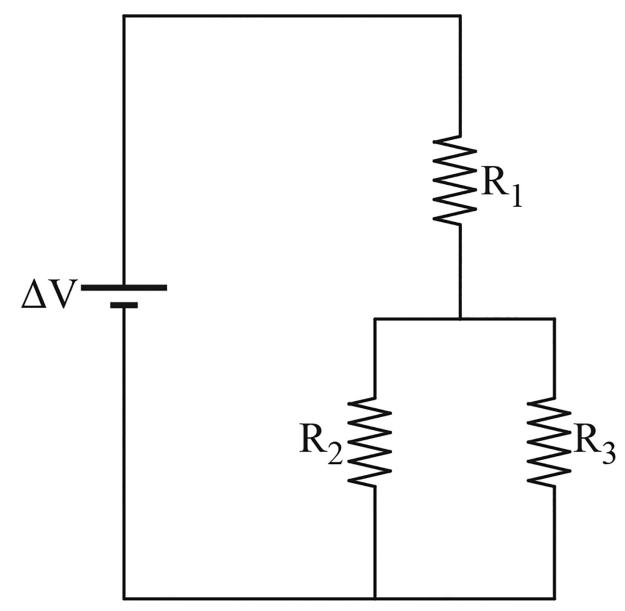

Figura 2: Circuito que resolvieron los estudiantes durante la actividad de andamiaje cognitivo. la batería (R1). Los estudiantes se dan cuenta de que como no conocen los valores de las resistencias, no pueden determinar que el equivalente de la conexión en paralelo sea menor que la resistencia en serie. A partir del conocimiento básico de "la resistencia equivalente debe ser menor que estas dos [R2 y R3]" El estudiante Bb concluyó que: "no sabemos cuál es mayor que cual, o sea no podemos saber si son iguales". Después continuaron relacionando las resistencias con los voltajes, y discutiendo sobre el tipo de aseveraciones que pueden hacer respecto al voltaje sin conocer las resistencias. El estudiante Ba concluyó que: "el voltaje de R2 es igual a R3" justificando que "están en paralelo". Reconocieron que los voltajes de las resistencias conectadas en paralelo deben de ser iguales.

Los estudiantes plantearon que el voltaje de R2 y R3 debía de ser menor que el de R1, sin embargo, tras una breve discusión se dieron cuenta que no podían asumir eso. Bb planteó la siguiente refutación: "Pero qué pasa si, por ejemplo, R2 y R3, la equivalente es mayor que R1, o sea que R2 y R3 son mucho mayores que R1". Después reconocieron que la batería es el límite del voltaje que puede tener el circuito. Ba lo justificó diciendo: "como están conectados a la misma batería, la suma de estas no puede ser mayor a ésta".

El equipo continuó la discusión sobre cómo el voltaje de la batería se debía de dividir. Uno de los estudiantes dijo que si había dos resistencias se dividía a la mitad, si había tres resistencias se dividía en tercios. Otro estudiante le respondió: "estás diciendo que la caída de voltaje depende de la resistencia, y no del valor de la resistencia, del número de resistencias", expresando que no está de acuerdo con eso. Mencionó que la corriente que pasa por R1 es la misma que pasa por el equivalente de R2 con R3. Regresó al caso que expuso anteriormente donde la resistencia equivalente de R2 y R3, que están conectadas en paralelo, fuera mayor que R1. Terminó su argumento diciendo que la equivalente de R2 y R3 es menor al menor, pero no necesariamente menor que R1.

Tras el extenso debate, el equipo comenzó a interactuar con otro equipo de la misma mesa para comparar sus resultados. El segundo equipo había llegado a la conclusión de que el voltaje en R1 es mayor que el voltaje en R2, que es igual al voltaje en R3. Cuestionaron esa respuesta y consultaron con el profesor. El profesor les preguntó qué tendría que pasar para que esa respuesta fuera cierta, y si tenían esa información. Entonces concluyeron que no podían establecer la relación de V1 con V2 ni de V1 con V3 ya que no conocían el valor de las resistencias.

En este argumento, los estudiantes pasaron por cuatro momentos clave para llegar a una conclusión: los estudiantes debían darse cuenta de que las resistencias no son iguales, recurrir a los tipos de conexiones que había entre las resistencias para encontrar sus relaciones, analizar las resistencias equivalentes de la conexión en paralelo y del sistema y reconocer que los voltajes de las 
resistencias no pueden ser mayores que el de la batería. En general en las discusiones observadas durante las actividades de andamiaje cognitivo se aprecia que los estudiantes necesitan incluir varios conceptos para llegar a una conclusión.

Las preguntas conceptuales generan debates donde se observan ciclos argumentativos elaborados que involucran todos los elementos primarios y secundarios del modelo de Toulmin para establecer una conclusión. Pueden existir diferentes posturas ante el tema, cambios de sus posturas iniciales y reconocimiento de supuestos. Las discusiones observadas durante las actividades de andamiaje cognitivo proveen de una guía que funciona como andamiaje en la construcción de conceptos. Por medio de la argumentación y la guía correcta, el estudiante puede utilizar un modelo y construir explicaciones basadas en ese modelo, el cual puede utilizar en distintos contextos [56].

\section{Discusión}

Para abordar los objetivos de investigación, se buscaron momentos donde, por medio de la argumentación, los estudiantes se enfrentaron a sus propias concepciones e ideas previas en el tema de circuitos eléctricos en un ambiente de aprendizaje centrado en el estudiante aplicando las estrategias didácticas de interés. Para responder el objetivo (1), los momentos de argumentación identificados se analizaron con el modelo de argumentación de Toulmin como marco de referencia. Para abordar el objetivo (2), se exploró cómo estos momentos contribuyen al aprendizaje de los estudiantes desde una perspectiva cualitativa. Para cumplir el objetivo (3), se destacan las diferencias entre las dinámicas de argumentación que surgen al implementar la estrategia de instrucción por pares y actividades de andamiaje cognitivo.

En los resultados presentados durante la instrucción por pares y en las actividades de andamiaje cognitivo, se puede observar que los estudiantes tienen dificultades y concepciones previas que, por medio de la argumentación, llegan a modificar o superar para llegar a una conclusión correcta. En instrucción por pares se observó que los estudiantes pasaron de elaborar argumentos sencillos (y correctos) a desarrollar razonamientos más complejos. Argumentos sencillos se refiere al uso de elementos básicos en el modelo de argumentación de Toulmin, mientras los argumentos más elaborados incluyen, además, elementos secundarios. Aumentar la complejidad del argumento implica que se cometieron errores en las conclusiones, pero los compañeros intervinieron por medio de la discusión para lograr superar esos errores y llegar a una conclusión correcta. En el caso expuesto, el estudiante involucró la diferencia de potencial en el modelo de corriente y resistencia. La capacidad de involucrar varios conceptos en el mismo modelo sugiere que el estudiante comprende con mayor profundidad estos temas.
Durante las actividades de andamiaje cognitivo, se observó que los estudiantes desarrollan la comprensión de circuitos eléctricos por medio de la argumentación. En el argumento que se expone, un estudiante afirmó que, si había dos resistencias, la corriente del circuito se dividía a la mitad, si había tres resistencias se dividía en tercios, a lo que su compañero respondió que no estaba de acuerdo porque estaba tomando en cuenta el número de resistencias y no su valor. El razonamiento que puede llevar a un estudiante hacia esta aseveración es suponer que las resistencias son iguales, sin embargo, en este ejercicio los estudiantes no conocían los valores de las resistencias. Teniendo esta retroalimentación directa de uno de sus compañeros, el estudiante puede reconocer que solamente si las resistencias son iguales puede establecer esas relaciones, pero si no se conoce el valor de las resistencias no se pueden establecer. Esto conlleva a que los estudiantes dejen de memorizar reglas [57], y se enfrenten a analizar cada circuito por separado utilizando razonamiento a nivel macroscópico, en términos de corriente y resistencia, o bien a nivel microscópico considerando el movimiento de las cargas [22].

En ambas estrategias didácticas se observó que los estudiantes desarrollan argumentos complejos y que todos los elementos giran alrededor de las conclusiones, lo que concuerda con el modelo de Toulmin [13, 23. Sin embargo, existen diferencias en cuanto a cómo suceden las discusiones. Una de las principales diferencias es que, durante la instrucción por pares, la dinámica de discusión se concentra en la mesa, mientras en las actividades de andamiaje cognitivo se concentra en los equipos pequeños.

En la sala de aprendizaje centrado en el estudiante, la cual es un ambiente estilo SCALE-UP, se había observado que las mesas funcionan como comunidades de aprendizaje donde los estudiantes trabajan en sus equipos pequeños e interactúan con el resto de la mesa para comparar o enriquecer su discusión. La dinámica de discusión que ocurre durante las actividades de andamiaje cognitivo corresponde con lo observado. En contraste, la dinámica que se da durante la instrucción por pares es distinta porque la argumentación se concentra entre varios estudiantes de los tres equipos que conforman la mesa, creando así una sola discusión en la comunidad de aprendizaje. Los integrantes que aportan elementos argumentativos de cada equipo pequeño forman el núcleo de la discusión, mientras el resto de los integrantes participan ocasionalmente o escuchan activamente la discusión.

Aun cuando el docente pide en ambas estrategias que las discusiones sean en pequeños grupos, los estudiantes deciden hacerla en la mesa completa. Una razón por la cual podría estar sucediendo esta diferencia entre estrategias, es que, en el caso de las actividades de andamiaje cognitivo, se promueve interdependencia positiva por medio de recursos. Esto quiere decir que los estudiantes obtienen una actividad impresa (pauta) por pequeño grupo, lo que los limita a trabajar con sus 
compañeros de equipo para desarrollar la tarea específica de la actividad. Durante la instrucción por pares esta restricción no existe, pues la pregunta que se analiza se proyecta en toda la sala, por lo que el límite espacial está dado por la mesa en el salón.

El método de instrucción por pares fue diseñado por Mazur y Hillborn [28] en un ambiente tradicional estilo auditorio, y los estudios que se han presentado sobre el análisis de sus argumentos también se ubican en este tipo de ambiente 35. En esta sala de aprendizaje centrado en el estudiante se ha utilizado la estrategia de instrucción por pares desde sus inicios; sin embargo, la exploración de los argumentos y la dinámica que se da durante esta estrategia en el ambiente de aprendizaje centrado en el estudiante no se había investigado. Es importante explorar el mismo método en ambos ambientes porque la instrucción por pares se usa en grupos masivos, del orden de cientos de estudiantes por salón. En cambio, los ambientes de aprendizaje centrado en el estudiante pueden acomodar a decenas o centenas de estudiantes, dependiendo del tamaño de la sala, pero cada mesa conforma una comunidad de aprendizaje de nueve personas [47. Los resultados presentados al utilizar la estrategia de instrucción por pares dan un panorama general acerca de las interacciones y la dinámica que se da en un ambiente estilo SCALE-UP.

\subsection{Conclusiones e implicaciones para la enseñanza de la ciencia}

La presente investigación tuvo como objetivo explorar las dinámicas de argumentación de los estudiantes al aprender circuitos eléctricos en un curso introductorio de electricidad y magnetismo que se lleva a cabo en un ambiente de aprendizaje centrado en el estudiante, donde se implementan estrategias de instrucción por pares y actividades de andamiaje cognitivo. El principal hallazgo de la investigación es que implementar estrategias que promueven la argumentación origina que los estudiantes logren un entendimiento conceptual profundo de los temas de circuitos eléctricos y desarrolla su capacidad para analizar y razonar el comportamiento de cada circuito en lugar de aplicar reglas memorizadas [22]. Este resultado contribuye a la investigación del aprendizaje de circuitos eléctricos por medio de aprendizaje activo [3, 22.

Las implicaciones para la enseñanza que resultan del presente estudio son acerca de la argumentación en relación con las estrategias didácticas y el espacio educativo donde se implementan. Por una parte, es importante destacar que la argumentación tiene un rol fundamental en las estrategias de instrucción por pares y actividades de andamiaje cognitivo. En ambas estrategias se observa que el estudiante es capaz de convertir sus ideas alternativas en conceptos que se adaptan mejor a los modelos científicos de electricidad y magnetismo, por medio de las discusiones con sus compañeros y los instructores. Cuando los estudiantes tienen la oportunidad de discutir y argumentar sobre los conceptos de clase, tienen un espacio para contrastar ideas y ajustar sus propias concepciones [51].

También se observó que el espacio educativo donde se implementan las estrategias didácticas que promueven la argumentación es importante en las dinámicas de discusión que ocurren de forma natural. Es importante considerar que hay espacios que promueven la argumentación entre pares. Mientras en espacios estilo auditorio, el centro de atención se encuentra sobre el profesor, en espacios estilo SCALE-UP, el centro de atención se ubica en el estudiante. Implementar esta idea en la práctica educativa implica repensar los espacios en los que sucede el aprendizaje y modificarlos, según los recursos disponibles.

Las limitantes del estudio tienen que ver con la naturaleza de la investigación, es decir, es un estudio cualitativo con un número acotado de estudiantes observados que no permite generalizar los resultados a toda la población. Además, el ambiente de aprendizaje por medio de un espacio SCALE-UP y las estrategias utilizadas de aprendizaje activo tienen un papel importante en el resultado de la investigación. Sin embargo, los resultados no solo serán reproducibles con condiciones similares, sino que también se prevé que se obtengan resultados similares en ambientes donde se promueva la interacción y con estrategias que fomenten la argumentación.

Finalmente, se considera que diferentes estrategias didácticas resultan en diferentes niveles de argumentación. La argumentación puede suceder en cuatro diferentes niveles en un contexto científico: en la mente del científico, en un grupo de científicos, en la comunidad científica y en el público en general [13]. De manera análoga, se puede decir que, en el aula, la argumentación sucede en la mente del estudiante, en equipos pequeños, en la comunidad de aprendizaje y con el grupo completo [18. Por medio de la combinación de instrucción por pares y las actividades de andamiaje cognitivo, se puede generar argumentación en estos cuatro niveles. En instrucción por pares, los estudiantes piensan individualmente y discuten con una comunidad de aprendizaje; en las actividades de andamiaje cognitivo, los estudiantes discuten en equipos pequeños. En ambas estrategias, los estudiantes confirman su conocimiento con el resto del salón, incluyendo los instructores.

\section{Agradecimientos}

Una de los autores, (S.T.) agradece al Proyecto DICYT, código 031931TF de la Universidad de Santiago de Chile, USACH.

\section{REFERENCIAS}

[1] L.C. McDermott y E.F. Redish, American Journal of Physics 67, 755 (1999).

[2] M. Cavinato, M. Giliberti y S.R. Barbieri, European Journal of Physics 38, 055707 (2017). 
[3] J.S. Flores y J. Benegas, Enseñanza de las ciencias: revista de investigación y experiencias didácticas 26, 245 (2008).

[4] C. Singh, American Journal of Physics 70, 1103 (2002).

[5] M.H. Bhuyan y S.S.A. Khan, International Journal of Learning and Teaching 10, 137 (2018).

[6] L.F. León, J.E. Duarte y F.H. Morales, Revista de investigación, desarrollo e innovación 4, 138 (2014).

[7] S. Tecpan, J. Benegas y G. Zavala, Latin-American Journal of Physics Education 9, 4 (2015).

[8] P. Barniol, E. Campos y G. Zavala, Enseñanza de las ciencias: revista de investigación y experiencias didácticas 36, 165 (2018).

[9] E. Bilal y M. Erol, Latin American Journal of Physics Education 3, 193 (2009).

[10] H. Peşman y A. Eryılmaz, The Journal of Educational Research 103, 208 (2010)

[11] E. Taşlıdere, Creative Education 4, 273 (2013).

[12] Y. Bogar, International Education Studies 12, 1 (2019).

[13] R. Driver, P. Newton y J. Osborne, Science Education 84, 287 (2000).

[14] J. Osborne, Science 328, 463 (2010).

[15] OCDE, PISA 2018 Results (Volume I): What Students Know and Can Do (OECD Publishing, Paris, 2019).

[16] E. Gaigher, J.M. Rogan y M.W.H. Braun, International Journal of Science Education 29, 1089 (2007)

[17] J. Benegas y H. Alarcón, en: El aprendizaje activo de la Física Básica Universitaria, editado por J. Benegas, M.C. Pérez de Landazábal y J. Otero (Andavira, Santiago de Compostela, 2013).

[18] E. Campos, L. Silva, S. Tecpan y G. Zavala, en Physics Education Research Conference, Sacramento, 2016, editado por D.L. Jones, L. Ding y A.L. Traxler (American Institute of Physics, Sacramento, 2016).

[19] M.H. Bhuyan, Journal of Bangladesh Electronics Society 14, 83 (2014).

[20] L.C. McDermott, American Journal of Physics 59, 301 (1991).

[21] F.A.L.D. Andrade, G.F. Barbosa, F.L.D. Silveira y C.A.D. Santos, Revista Brasileira de Ensino de Física 40, e3406 (2018).

[22] A. Leniz, K. Zuza y J. Guisasola, Physical Review Physics Education Research 13, 010128 (2017).

[23] M. Jiménez-Aleixandre, V. Alvarez Pérez y J. Lago Lestón, Tarbiya, Revista de Investigación e Innovación Educativa 36, 35 (2005).

[24] S.E. Toulmin, The uses of argument (Cambridge University Press, Cambridge, 2003).

[25] O. Molina, V. Font y L. Pino-Fan, Enseñanza de las ciencias 37, 93 (2019).

[26] A. Bertelle, A. Rocha y J.M. Domínguez Castiñeiras, Revista Eureka sobre Enseñanza y Divulgación de las Ciencias 11, 114 (2014)

[27] I. García-Rodeja Gayoso y V. Sesto Varela, Revista Eureka sobre Enseñanza y Divulgación de las Ciencias 13, 215 (2016)

[28] E. Mazur y R.C. Hilborn, Physics Today 50, 65 (1997).
[29] G. Zavala, en: Upgrading Physics Education to Meet the Needs of Society, editado por M. Pietrocola (Springer International Publishing, Basel, 2019).

[30] M. Prince, Journal of Engineering Education 93, 223 (2004).

[31] K. Hogan, B.K. Nastasi y M. Pressley, Cognition and Instruction 17, 379 (1999).

[32] C. Singh, American Journal of Physics 73, 446 (2005).

[33] P. Zhang, L. Ding y E. Mazur, Physical Review Physics Education Research 13, 010104 (2017).

[34] A.V.R. Araujo, E.S. Silva, V.L.B. Jesus y A.L. Oliveira, Revista Brasileira de Ensino de Física 39, e2401 (2017).

[35] C.H. Crouch y E. Mazur, American Journal of Physics 69, 970 (2001).

[36] J. Mackiewicz y I. Thompson, Composition Studies 42, 54 (2014).

[37] D. Wood, J.S. Bruner y G. Ross, Journal of Child Psychology and Psychiatry 17, 89 (1976).

[38] L. Ding, N. Reay, A. Lee y L. Bao, Physical Review Special Topics-Physics Education Research 7, 020109 (2011).

[39] A. Garza y G. Zavala, en: Physics Education Research Conference, Portland, 2010, editado por M. Sabella, C. Singh y N. Rebello (American Institute of Physics, Melville, 2010).

[40] R. Leinonen, M.A. Asikainen y P.E. Hirvonen, Physical Review Special Topics-Physics Education Research 9, 020112 (2013).

[41] S. Lin y C. Singh, Physical Review Special TopicsPhysics Education Research 11, 020105 (2015).

[42] C. Lisdstrom y M.D. Sharma, Physical Review Special Topics-Physics Education Research 7, 010109 (2011)

[43] I. Roll, N.G. Holmes, J. Day y D. Bonn, Instructional Science 40, 691 (2012).

[44] C. Singh, Physical Review Special Topics-Physics Education Research 4, 010105 (2008).

[45] L.C. McDermott y P.S. Shaffer, Tutoriales para física introductoria (Pearson Education, Buenos Aires, 2001).

[46] J. Guisasola, M. Garmendia, A. Montero y J. I. Barragués, Enseñanza de las Ciencias 30, 61 (2012).

[47] G. Zavala, A. Domínguez y R. Rodríguez, en: Enfoques en Investigación e Innovación en Educación: Vol. 1. Innovación Educativa, Educación Superior y Formación Docente, editado por A. Domínguez (REDIIEN, Monterrey, 2014).

[48] S.B. Merriam, Qualitative research: A guide to design and implementation (Jossey-Bass, San Francisco, 2009).

[49] H. Young y R. Freedman, Física Universitaria con Física Moderna (Pearson Educación, México, 2013), 13. ${ }^{a}$ ed., v. 2.

[50] M. Micari y D. Drane, Active Learning in Higher Education 12, 175 (2011).

[51] C.J. Ballen, C. Wieman, S. Salehi, J.B. Searle y K.R. Zamudio, CBE - Life Sciences Education 16, ar56 (2017).

[52] D.A. Erlandson, E.L. Harris, B.L. Skipper y S.D. Allen, Doing naturalistic inquiry: A guide to methods (Sage Publications, Newbury Park, 1993). 
[53] M.A. Spillett, Academic Exchange Quarterly 7, 36 (2003).

[54] P.V. Engelhardt y R.J. Beichner, American Journal of Physics 72, 98 (2004).

[55] E. Brewe, L. Kramer y V. Sawtelle, Physical Review Special Topics-Physics Education Research 8, 010101 (2012).

[56] J. Osborne, Thinking Skills and Creativity 10, 265 (2013).

[57] A. Madsen, S.B. McKagan y E.C. Sayre, Physical Review Special Topics-Physics Education Research 11, 010115 (2015). 